Research/Technical Note

\title{
Joint Functional Screening (JFS): Movement for Optimal Health and Body Function: Perspective Clinical Examination
}

\author{
Balwant Singh Bains \\ Department of Physiotherapy, Aihmsa College, Kuala Lumpur, Malaysia
}

Email address:

datobains@gmail.com

\section{To cite this article:}

Balwant Singh Bains. Joint Functional Screening (JFS): Movement for Optimal Health and Body Function: Perspective Clinical Examination. Rehabilitation Science. Vol. 3, No. 1, 2018, pp. 13-16. doi: 10.11648/j.rs.20180301.13

Received: January 9, 2018; Accepted: January 25, 2018; Published: February 12, 2018

\begin{abstract}
Background: Body's locomotor system, at any given age, need to be pain-free, agile, stable and energetic, symmetrical in joint functional system and muscle power. Joint Functional Screening (JFS) is a musculo-skeletal profiling program via a systematic clinical examination with clinical reasoning of the entire human joints' functional system, with or without causative limitation to derive a holistic analysis to give a health provider, the strength, flexibility, and stability indicators of the musculoskeletal system. Objective: The purpose of this study was to introduce a feasible and an affordable clinical examination package as Joint Functional Screening. Method: This package including clinical tools to analysis and screen all body joints which can determine muscular imbalances and body abnormalities. Conclusion: JFS is an innovation program to profile a normal musculoskeletal state to decode any underlying anomalies of the body, in an otherwise normal subject, who might be preparing to take up any sporty or gym activities in one's lifespan that could elicit or uproot an injury which could be otherwise prevented.
\end{abstract}

Keywords: Musculoskeletal, Joint Functional Screening (JFS), Health, Body Function

\section{Introduction}

Human body changes year after year. The bone formation \& joint structures, muscle action, the organs, the chemistry and not forgetting the firing of the nervous system keeps the body alive. Again it is subjected to the requirement of mankind and the environment we live in and by increasing the age, the changes in connective tissue and cartilage that has detrimental effect on joint [1].

The joint's cartilage becomes more tenuous and lead to change the cartilage and ligaments components, which can create the joint become stiffer, develop limitation in range of motion and become more prone to injuries [2] [3]. With increased age, neuromuscular dysfunction could occur due to many other conditions. A few to mention, are like, overweight, low in physical activities, bad posture of sitting, standing and sleeping as well [4]. The ankle, knee, hip and low back pain is a common symptom of an imbalance body alignment; these joints are designed to undergo repeated motion and facilitate fluid movement. Many factors can contribute to those pains; apart from above mentioned are also like, injuries, lack of flexibility, heavy lifting causing repetitive injuries at work place, and improper sleeping positions and poor bedding conditions that could elicit pain in an otherwise unbalanced body [5]. However, because of an imbalance body alignment there can be added stress to the whole body and joint structures that can cause a dysfunctional pain in the joint, ligaments, tendons, bursa, and related muscles. This will incapacitate daily activities like walking, working or during activities of daily living. In addition, the feet are the foundation of entire body for ambulation purpose and help to support body weight and act as shock absorbers [6]. Imbalance foundation also may increase risk of falling among healthy individuals. Hence, musculoskeletal stability and core stability properties of antagonistic muscles support the stability for cyclic movements and balance [7]. Investigating and screening muscle strength, flexibility, balance, and body alignment of human body can prevent injuries during activity during the lifespan [8-10]. 
The rate of injuries such as in daily domestic or workplace repetitive activities, exercise regime, sports, school activities, jogging and gym exercises may arise because of imbalance body alignment and musculoskeletal disorder [11, 12] are in the rise. The risk of fall injuries increases because of gait changes that bring about instability, bad posture and loss of balance among elderly people [13]. Falls are often caused by changes in the muscles imbalance and hence screening the joint and muscles can provide a personal profile to avoid any injuries and musculoskeletal problem [14].

Professional rehabilitative clinic currently are only performing specific isolated, objective testing for specific muscles and joints only. Moreover, some clinicians and physical therapist frequently perform clinical rehabilitation and intervention following specific skill assessments of the area of concern by the patient without correlating functional movements of the adjacent joints and muscles. It is important to screen and identify general fundamental parts of human movements, joints, and muscles dealing throughout a complex activity. The professional rehabilitation centers must appreciate that in order to prepare individual's rehabilitation for every type of activities, screening of fundamental associate movements is essential. Previous studies still rely on screening one joint independently. For example, one study [6] of screening body index and foot flatness, attention to all other related part of body is not taken into account as a package of information. Based on our knowledge and search, there is a lack in the entire human body joints' functional screening, (in one format), thus reflecting a gap to pave an innovation in designing a new methodology of a profiling system suitable for any individual who are otherwise healthy. This paper introduces the new package of Joint functional screening, which is more feasible and an affordable clinical examination.

\subsection{Application of JFS}

JFS should be adopted in the practice of:

1. Sports selection board - JFS would provide guidelines and empowerment to athletes and coaches

2. Military selection board - JFS would provide guidelines of the suitability of the selected person to carry out vigorous training. The current routine clinical examination in the military, to measure the height, weight, eye-sight and the state of the cardinal signs may not be enough to provide the absolute profile of a body.

3. School and teenager: JFS could become a perfect and reliable tool used to monitor the growth of an individual, and identify growth abnormality and provides useful guidelines for healthcare centers when physical activity among adolescents and children increased.

4. Office Ergonomics - Corporates where standing and walking is required throughout the workplace and prolonged sitting at the office computer work station. JFS assessment can assist the corporate to reduce and prevent anticipated medical conditions that would help to reduce the medical budgets on conditions affecting the joints due to repetitive stress situations.

5. At gyms - Before any gym program, JFS could become an important tool as guidance to the Gym Instructors. This would avoid any untoward injuries during the regime at the gym.

\subsection{Purpose of Joint's Functional Screening (JFS)}

1. To perform standardized training to all therapists to carry out musculoskeletal management protocol, analysis and reporting to enhance the wellness of an individual.

2. Used as an important tool to determine pre-requisite muscle performance and conditioning before sport, military or martial arts, wellness center programs and exercises tailored for lifestyle diseases.

3. To design a tailor-made exercise regime for all individuals and objectivize the progress after re-evaluation using JFS methodology. Hence it acts as a baseline for measurements as well as to determine improvement in muscle performance in the follow-up analysis.

4. To provide the therapist a measure to prescribe the appropriate intervention to develop muscles strength, endurance and performance.

\section{Procedure}

\subsection{Observation or Orientation Phase}

This is the phase when a therapist observes the clinical examination carried out by an accredited Examiner. Before examination the concepts and application of JFS were explained to the participants. Prior to the examination, the participants fill up the consent form.

In this phase, the therapist is exposed to the methodology learn and understand systematic examination and uniformity of assessment based on a standardized approach. This helps them to avoiding bias errors through use of multiple movement parameters, to obtain fairer assessment. This is in view of standardizing the assessment techniques of all the joints when the client or patients are advised, recommended or prescribed an intervention JFS program. The Observer shall have to understand the positioning, precaution and comfort in designing the type and intensity of stretching and the type of resistance used. During this phase, there is written exam on questions that the examiner may pose while observation. Number of hours a therapist observes the examination on various subject is noted in a log-book designed for the purpose.

\subsection{Data Entry and Note-Taking Phase}

The observer then advances to this phase who has now enough knowledge of the protocols used to carry out the examination which are learnt during the observation phase. In this phase, the therapist exposes to mark indicators for the number of stretching and resisted exercises; the intensity of stretching and the repetition of resistance exercises. During this phase, the therapist is able to design a tailor-made exercises program for the participant. 


\subsection{Examination Phase}

This is the hands-on phase. The therapists become an examiner. The therapist now duplicates all the procedure learned during the orientation and data-entry phase. The therapist ought to carefully listen to the history or medical condition narrated by the participants and record all data. Participants have to remove all the belongings from the pants including watches and spectacles. The examiners check the variables accordingly to table 1 . As the examination is carried out the narration of the finding is recorded by another therapist who is on training in the Note-Taking phase. At the end of screening the examiners along with the therapist on data entry would analysis the data and according to results of screening, exercise protocol are designed and recommended.

Table 1. Variables and Measurements Tools.

\begin{tabular}{|c|c|}
\hline Variables & Measurement tools \\
\hline Upper body strength & Physical examination modified oxford scale \\
\hline Lower body strength & Chair Sit-to-Stand Test to measure the quadriceps and hamstring \\
\hline Hip Flexibility & Straight Leg Raise (SLR) Test \\
\hline Trunk Flexibility & Sit and Reach Flexibility Test Trunk Rotation Test \\
\hline Knee Flexibility & Active Knee Extension Hamstring Flexibility test \\
\hline Lordosis & Wall Test \\
\hline Scoliosis & Adam's forward bend test \\
\hline Tensor Fascia Lata (TFL) tenderness & Check the Symptoms of TFL Syndrome \\
\hline Body alignment and foot scanning & By Foot levelers machine \\
\hline Body mass index or body composition & In-Body Machine \\
\hline Balance & One leg stance test open and close eye) \\
\hline Spinal alignment & Plumb Line \\
\hline TA tightness & Thomas test \\
\hline Gait and functional mobility & 10 Meter walking test and TUG \\
\hline Check the leg length. & Measuring from the anterior superior iliac spine \\
\hline Genu Valgum & Plumb line \\
\hline Genu Varum & Plumb line \\
\hline Quards endurance Test & Sit to stand and hold test. \\
\hline
\end{tabular}

\section{Conclusion}

Joint functional screening is a program that could fit into a "missing block" as a prerequisite before a subject takes up any form of exercise or sport activities. Joints Functional Screening can become an asset to the world of sports and healthy living program as participants are empowered with the understanding of their own body structures; of their musculoskeletal uniformity; muscle performance before sports; military national activities and the list can go on. JFS also suggests appropriate entry point for safe exercises and enhance muscle strength, endurance, and flexibility. This study are useful for the physiotherapists, exercise therapist, personal trainers by screen body prior to beginning a rehabilitative and exercise program; and this screen package is the best guidance to prevent risk of injuries among individual healthy people. Moreover, this study has significance in community healthcare set up.

\section{References}

[1] Mosavat, M., Mirsanjari, M., Omar, S. Z., \& Sadeghi, H. (2014). Bone Health, Exercise and Nutrition. Journal of Advanced \& Applied Sciences, 2(4),143-156.

[2] Chen, H., Zhou, X., Fujita, H., Onozuka, M., \& Kubo, K. Y. (2013). Age-related changes in trabecular and cortical bone microstructure. International journal of endocrinology, 2013 (213234).
[3] Tsai, M. S., \& Hsu, A. T. (2015). The aging effects on the glenohumeral joint stiffness in association with anteroposterior glide mobilization: in vivo robotic testing. Physiotherapy, 101, e594.

[4] Shariat, A., Tamrin, S., Mohd, B., Arumugam, M., Danaee, M., \& Ramasamy, R. (2016). Prevalence rate of musculoskeletal discomforts based on severity level among office workers. Acta Medica Bulgarica, 43(1), 54-63.

[5] Shariat, A., Bahri Mohd Tamrin, S., Daneshjoo, A., \& Sadeghi, H. (2015). The adverse health effects of shift work in relation to risk of illness/disease: a review. Acta Medica Bulgarica, 42(1), 63-72.

[6] Chougala, A., Phanse, V., Khanna, E., \& Panda, S. (2015). Screening of body mass index and functional flat foot in adult: an observational study. Int J Physiother Res, 3(3), 1037-41.

[7] Wagner, H., Anders, C. H., Puta, C. H., Petrovitch, A., Mörl, F., Schilling, N., ... \& Blickhan, R. (2005). Musculoskeletal support of lumbar spine stability. Pathophysiology, 12(4), 257-265.

[8] Bains, B. S., Kaur, G., Sadeghi, H., Husain, A., \& Singh, K. (2016). Trunk Flexibility Improvement in Response to Powered Assisted Exercise. International Journal of Kinesiology \& Sports Science, 4(3), 50-53.

[9] Cook, G., Burton, L., Hoogenboom, B. J., \& Voight, M. (2014). Functional movement screening: The use of fundamental movements as an assessment of function-Part 2. International journal of sports physical therapy, 9(4), 549.

[10] Knapik, J. J., Cosio-Lima, L. M., Reynolds, K. L., \& Shumway, R. S. (2015). Efficacy of functional movement screening for predicting injuries in coast guard cadets. The Journal of Strength \& Conditioning Research, 29(5), 1157-1162. 
[11] Cantley, L. F., Taiwo, O. A., Galusha, D., Barbour, R., Slade, M. D., Tessier-Sherman, B., \& Cullen, M. R. (2014). Effect of systematic ergonomic hazard identification and control implementation on musculoskeletal disorder and injury risk. Scandinavian journal of work, environment \& health, 40(1), 57.

[12] Piper, S., Shearer, H. M., Côté, P., Wong, J. J., Yu, H., Varatharajan, S.,... \& Nordin, M. C. (2016). The effectiveness of soft-tissue therapy for the management of musculoskeletal disorders and injuries of the upper and lower extremities: a systematic review by the Ontario Protocol for Traffic Injury management (OPTIMa) collaboration. Manual therapy, 21, 18-34.

[13] Sadeghi, H., Amri, S. B., Razeghi, M., Hamid, T. A., \& Abdollah, M. N. H. (2017). Effects of Combined exergame and conventional exercise to reduce and prevent fall risk among elderly people: A Hypothesis. International Journal of Applied Exercise Physiology, 6(3), 80-84.

[14] Laughton, C. A., Slavin, M., Katdare, K., Nolan, L., Bean, J. F., Kerrigan, D. C.,... \& Collins, J. J. (2003). Aging, muscle activity, and balance control: physiologic changes associated with balance impairment. Gait \& posture, 18(2), 101-108.

\section{Biography}

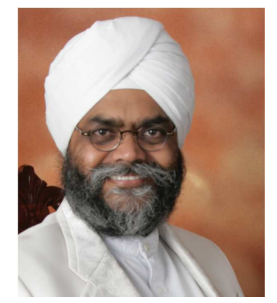

Dato' Dr. Balwant Singh Bains, Chief Senior Consultant Physiotherapist, BainsPhysio Group Practice.Dato' Dr. Bains is a physiotherapist with 30 years in clinical practice and health care business. Founder of BainsPhysio ${ }^{\circledR}$ (1985), Aihmsa College 2011 (an Institute of Health Care, Movement Science \& Arts). He attained a $\mathrm{PhD}$ in Psychology in 1997. His interest is in Musculoskeletal Area and has been involved in well accepted Medico-legal quantum justification to legal firms. Authorship Licensor for "Joints' Functional Screening"- A profiling system of the musculo-skeletal state before performing sports and gym activities, geriatric exercises and post $\&$ pre musculo skeletal surgery. 